\title{
Evaluación y comparación del desempeño de cinco modelos de predicción de mortalidad intrahospitalaria en pacientes con falla cardiaca aguda
}

\author{
Evaluation and comparison of the performance of five prediction models of in-hospital \\ mortality in patients with acute heart failure
}

\author{
Marietta Juan-Guardela, Ángel García-Peña*, Alejandro Mariño-Correa, Emilio Juan-Guardela, \\ Lina Arbeláez, Lauren Calvo y Margarita Valencia \\ Pontificia Universidad Javeriana, Hospital Universitario San Ignacio, Bogotá, Colombia
}

\section{Resumen}

Objetivo: Los modelos de predicción de mortalidad intrahospitalaria en pacientes con falla cardiaca aguda pueden ser útiles para la toma de decisiones, situación que hace necesario evaluar la capacidad predictiva y de discriminación en la población colombiana. Método: Estudio de cohorte retrospectiva de pacientes con falla cardiaca aguda. Se evaluó el desempeño de los modelos de predicción de mortalidad intrahospitalaria ADHERE, OPTIMIZE-HF, GWTG-HF y PROTECT, durante los años 2013 a 2015. Se realizó el cálculo de la puntuación para cada uno de los modelos y se determinó la capacidad de predicción y discriminación. Resultados: Se incluyeron 776 pacientes con una edad promedio de 71.5 años (desviación estándar: 14.3), el $56 \%$ hombres, con fracción de eyección del ventrículo izquierdo del 39\%. La mortalidad global fue del 6.1\%. El área bajo la curva para ADHERE fue de 0.56 (intervalo de confianza del 95\% [IC95\%]: 0.49-0.64), para EHMRG de 0.63 (IC95\%: 0.550.71], para GWTG-HF de 0.63 (IC95\%: 0.55-0.70), para OPTIMIZE de 0.65 (IC95\%: 0.56-0.74) y para PROTECT de 0.69 (IC95\%: 0.60-0.77). Conclusiones: Los modelos de predicción de muerte intrahospitalaria en pacientes con falla cardiaca aguda muestran pobre desempeño y baja capacidad de predicción y discriminación en población colombiana, lo cual sugiere el desarrollo de escalas de predicción de mortalidad en pacientes con falla cardiaca aguda específicas para dicha población.

Palabras clave: Mortalidad. Falla cardiaca. Modelos de riesgo.

\section{Abstract}

Objective: In-hospital mortality prediction models on acute heart failure can be beneficial for decision-making, a situation necessary to evaluate, our goal was to compare predictive and discriminatory capacity of Colombian population. Method: $A$ retrospective cohort study in patients with acute heart failure was conducted. The following performance evaluation of in-hospital mortality prediction models were conducted from 2013 to 2015: ADHERE, EHMRG, OPTIMIZE-HF, GWTG-HF and PROTECT. Data was calculated for each model, prediction and discriminatory capacity was evaluated. Results: $A$ sample of

Correspondencia:

*Ángel García-Peña

E-mail: aagarcia@husi.org.co
Fecha de recepción: 21-11-2019

Fecha de aceptación: 31-07-2020

DOI: 10.24875/RCCAR.M21000052
Disponible en internet: 29-07-2021 Rev Colomb Cardiol. 2021;28(3):269-273 www.rccardiologia.com 0120-5633 / @ 2020 Sociedad Colombiana de Cardiología y Cirugía Cardiovascular. Publicado por Permanyer. Este es un artículo open access bajo la licencia CC BY-NC-ND (http://creativecommons.org/licenses/by-nc-nd/4.0/). 
776 patients, 56\% male, with an average age of 71.5 (standard deviation: 14.3) and with left ventricle ejection fraction rate of $39 \%$ was studied. Global mortality was of $6.1 \%$. The area under curve for ADHERE was of 0.56 (95\% confidence interval [95\% Cl]: 0.49-0.64), for EHMRG 0.63 (95\%Cl: 0.55-0.71), for GWTG-HF 0.63 (95\% Cl: 0.55-0.70), for OPTIMIZE 0.65 (95\%Cl: 0.56-0.74) and for PROTECT 0.69 (95\% Cl: 0.60-0.77). Conclusions: The models for predicting in-hospital death in patients with acute heart failure show poor performance, predictability and discrimination in the Colombian population, suggesting the development of mortality prediction scales in patients with acute heart failure specific to our population.

Key words: Mortality. Heart failure. Risk models.

\section{Introducción}

La falla cardiaca es considerada un problema de salud pública. Su prevalencia en población adulta oscila entre el $0.4 \%$ y el $2 \%$, y aumenta con la edad, ya que para los mayores de 70 años alcanza más del $10 \%$ y su incidencia es de aproximadamente 10 por 10,000 personas en los mayores de 65 años. En 2012, según el Ministerio de Salud y Protección Social de Colombia, la prevalencia de falla cardiaca en la población general era del $2.3 \%$, y de este porcentaje, el $59.7 \%$ eran hombres y el $40.3 \%$ eran mujeres ${ }^{1}$.

La mortalidad por falla cardiaca aguda viene en descenso en los últimos años. Esta se modifica por factores como la edad, la gravedad, el estadio, la función ventricular y el manejo recibido². En Colombia, la mortalidad durante la fase hospitalaria es de alrededor del $16 \%$, durante seguimiento a 3 meses es del $31 \%$, a 6 meses es del $37.6 \%$ y a 1 año es del $4.2 \%{ }^{3}$.

Los cuadros de descompensación aguda de esta enfermedad generan altos costos para el sistema de salud, derivados de las consultas a servicios de urgencias, las hospitalizaciones, el tratamiento farmacológico, la rehabilitación cardiaca y la discapacidad asociada'.

Por consiguiente, la estratificación del riesgo es una tarea médica esencial, lo cual está dirigido a detectar pacientes con condiciones de alto riesgo y probabilidad elevada de mortalidad o de reingreso, con el objetivo de intensificar las medidas de tratamiento y seguimiento para este grupo de pacientes.

Existen diferentes modelos de predicción del riesgo de mortalidad intrahospitalaria o de desenlaces adversos durante la hospitalización y hasta a 1 año desde el episodio. Entre ellos, los más utilizados son ADHE$\mathrm{RE}^{4}$, OPTIMIZE-HF ${ }^{5}$ y GWTG-HF${ }^{6}$, que se encuentran recomendados en las guías internacionales para el manejo de pacientes con falla cardiaca ${ }^{7}$ y algunos cuentan con validaciones en poblaciones diferentes de la cohorte inicial ${ }^{6}$; sin embargo, ninguno se utiliza sistemáticamente en nuestra institución y no existe, en el momento, una recomendación formal para su uso en las guías y los consensos para el manejo de la falla cardiaca en Colombia ${ }^{1,4-9}$.

Una estratificación del riesgo adecuada puede mejorar los desenlaces clínicos o evitar tanto el sobretratamiento de pacientes de bajo riesgo como el egreso hospitalario inapropiado de aquellos de alto riesgo. Para predecir la mortalidad durante la estancia hospitalaria y a corto plazo se han derivado y validado varios marcadores clínicos ${ }^{1}$. De acuerdo con la alta prevalencia de esta enfermedad y con que estos modelos pueden ser útiles para la toma de decisiones, el objetivo de este estudio fue evaluar y comparar su capacidad predictiva y de discriminación en población colombiana.

\section{Método}

Estudio de pruebas diagnósticas de una cohorte retrospectiva de pacientes adultos, admitidos en el servicio de urgencias del Hospital Universitario San Ignacio de Bogotá, con diagnóstico de falla cardiaca aguda, recolectadas entre enero de 2013 y diciembre de 2015. Se incluyeron pacientes adultos, con diagnóstico de falla cardiaca aguda y estancia hospitalaria mayor de 24 horas. Se excluyeron aquellos con antecedente de trasplante de cualquier órgano o de médula ósea, falla cardiaca avanzada en lista de espera de trasplante cardiaco o con dispositivos de asistencia ventricular de cualquier tipo, y remitidos a otra institución. Para la recolección de la información se utilizaron el programa de historia clínica electrónica de la institución y la lista de ingresos hospitalarios de enero de 2013 a diciembre de 2015, con diagnósticos de ingreso de insuficiencia cardiaca congestiva (1500), insuficiencia ventricular izquierda (1501) o insuficiencia ventricular izquierda (1509). Se excluyeron pacientes con diagnóstico al ingreso de falla cardiaca cuyo diagnóstico fue modificado o reemplazado tras la realización de evaluaciones.

De acuerdo con la naturaleza de cada variable, se describió la población con medidas de tendencia 


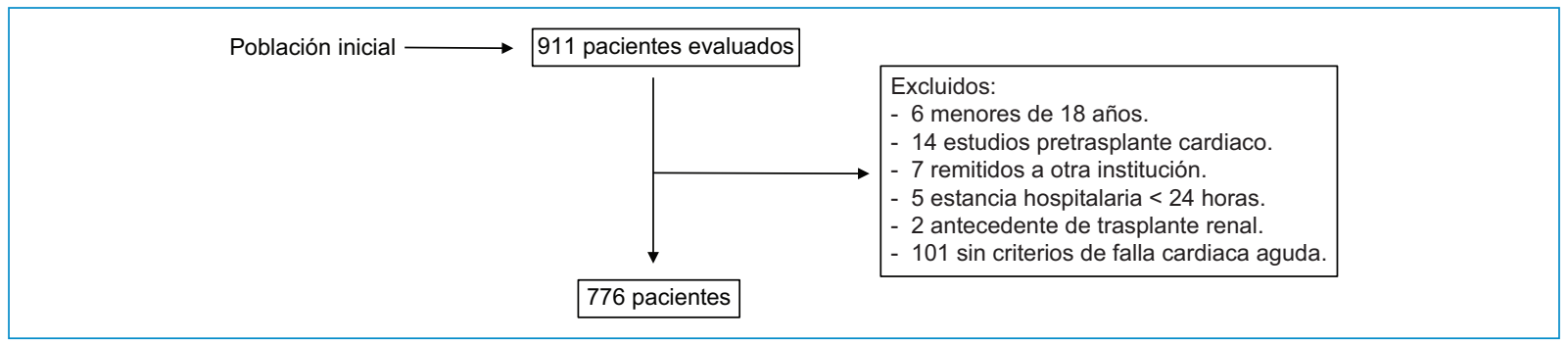

Figura 1. Obtención de la muestra.

central y de dispersión para las variables continuas y con frecuencias absolutas y relativas para las variables categóricas. Se utilizaron promedios y desviaciones estándar para las variables cuantitativas, en tanto que para las variables nominales y ordinales se emplearon la descripción de conteos, las frecuencias y los porcentajes. Posteriormente, se realizó el cálculo de cada uno de los índices de interés y se evaluó el número de eventos (esperados y presentados). Según el número de eventos, se realizó el análisis de la capacidad de discriminación de las escalas mediante evaluación a través de la comparación de las áreas bajo la curva ROC (Receiver Operating Characteristic) y cálculo de las características operativas para diferentes puntos de corte según los percentiles (OPTIMIZE, GWTG-HF) o la clasificación o predicción de riesgo (ADHERE, EHMRG9, PROTECT10). Se trabajó con un nivel de significancia $<0.05$ y un estadístico $C$ esperable de 0.7 .

El estudio fue aprobado por el comité de investigación y ética de la Facultad de Medicina de la Universidad Javeriana basándose en los principios éticos y científicos citados en la declaración de Helsinki, versión 64. ${ }^{\text {a }}$ Asamblea General, Fortaleza, Brasil, octubre de 2013.

\section{Resultados}

Entre enero de 2013 y diciembre de 2015 se reclutaron 911 pacientes, de los cuales se incluyeron 776 y se excluyeron 135 de acuerdo con los criterios establecidos (Fig. 1).

El $56 \%$ eran hombres y el $44 \%$ eran mujeres, con una edad promedio de 71.5 años (desviación estándar [DE]: 14.3). El tiempo promedio de estancia hospitalaria fue de 10 días y la mortalidad intrahospitalaria global fue del $6.18 \%$. En cuanto a los antecedentes, un $69 \%$ presentaba hipertensión arterial, un $63 \%$ falla cardiaca previa, un $30 \%$ enfermedad pulmonar obstructiva crónica, un 29\% fibrilación auricular, un 26\% enfermedad renal crónica y un $24 \%$ diabetes mellitus. Se estimó una fracción de eyección del ventrículo izquierdo promedio del 39.8\% (DE: 16.4\%), una presión arterial sistólica de $127.8 \mathrm{mmHg}$ (DE: $40.9 \mathrm{mmHg}$ ), una frecuencia cardiaca de 82.8 latidos por minuto (DE: 21.1 latidos por minuto), una frecuencia respiratoria de 20.3 respiraciones por minuto (DE: 4.9 respiraciones por minuto) y una saturación arterial de oxígeno del $91.5 \%$ (DE: $6.1 \%$ ). El $8 \%$ de los pacientes presentaron signos electrocardiográficos de isquemia y el $49 \%$ signos radiográficos congestivos.

El promedio de los valores del péptido natriurético cerebral fue de $2368 \mathrm{pg} / \mathrm{dl}$, el de creatinina de $1.48 \mathrm{mg} /$ dl y el de nitrógeno ureico en sangre de $29.1 \mathrm{mg} / \mathrm{dl}$. Las características generales de la población se describen en la tabla 1.

Al realizar el análisis de la capacidad de predicción de la mortalidad intrahospitalaria se encontró un área bajo la curva de 0.56 (intervalo de confianza del 95\% [IC95\%]: 0.49-0.64) para el ADHERE, de 0.63 (IC95\%: 0.55-0.71] para el EHMRG, de 0.63 (IC95\%: 0.55-0.70) para el GWTG-HF, de 0.65 (IC95\%: 0.56-0.74) para el OPTIMIZE y de 0.69 (IC95\%: 0.60-0.77) para el PROTECT (Figs. 2-3).

\section{Discusión}

En este estudio se evaluaron y compararon cinco modelos de predicción de muerte intrahospitalaria en pacientes con falla cardiaca aguda. Los resultados obtenidos indican una pobre precisión de la predicción de mortalidad con estas escalas, con un área bajo la curva entre $0.56(0.49-0.64)$ y $0.69(0.60-0.77)$. Se ha publicado un estudio en el cual se incluyeron pacientes hospitalizados con diagnóstico de falla cardiaca descompensada para evaluar la mortalidad intrahospitalaria mediante escalas de riesgo (ADHERE, OPTIMIZE y GWTG-HF) en un hospital de tercer nivel en 
Rev Colomb Cardiol. 2021;28(3)

Tabla 1. Características generales de la población

\begin{tabular}{|c|c|}
\hline Variable & Valor \\
\hline Edad (años) & 71.5 (DE: 14.3) \\
\hline Mujeres & $342(44 \%)$ \\
\hline Hombres & $434(56 \%)$ \\
\hline FEVI $(\%)$ & 39.8 (DE: 16.4) \\
\hline Presión arterial sistólica $(\mathrm{mmHg})$ & 127.8 (DE: 40.9) \\
\hline Frecuencia respiratoria (r.p.m.) & 20.3 (DE: 4.9) \\
\hline Frecuencia cardiaca (I.p.m.) & 82.8 (DE: 21.1) \\
\hline $\mathrm{SatO}_{2}(\%)$ & 91.5 (DE: 6.1 ) \\
\hline $\begin{array}{l}\text { Comorbilidad } \\
\text { Hipertensión arterial (\%) } \\
\text { Diabetes mellitus }(\%) \\
\text { Dislipidemia }(\%) \\
\text { Falla cardiaca previa (\%) } \\
\text { Fibrilación auricular (\%) } \\
\text { EPOC }(\%) \\
\text { Enfermedad renal crónica (\%) } \\
\text { Enfermedad arterial periférica (\%) } \\
\text { Cáncer }(\%)\end{array}$ & $\begin{array}{c}69 \\
24 \\
19 \\
63 \\
29 \\
30 \\
26 \\
4 \\
6\end{array}$ \\
\hline $\begin{array}{l}\text { Hallazgos } \\
\text { Electrocardiograma con signos de isquemia } \\
(\%) \\
\text { Radiografía de tórax con signos } \\
\text { congestivos }(\%)\end{array}$ & $\begin{array}{c}8 \\
49\end{array}$ \\
\hline $\begin{array}{l}\text { Paraclínicos } \\
\text { Péptido natriurético (pg/dl) } \\
\text { Hemoglobina }(\mathrm{g} / \mathrm{dl}) \\
\text { Creatinina }(\mathrm{mg} / \mathrm{dl}) \\
\text { BUN }(\mathrm{mg} / \mathrm{dl}) \\
\text { Sodio }(\mathrm{mEq} / \mathrm{l}) \\
\text { Potasio }(\mathrm{mEq} / \mathrm{l}) \\
\text { Glucemia }(\mathrm{mg} / \mathrm{dl})\end{array}$ & $\begin{array}{c}2368 \text { (DE: } 3672 \text { ) } \\
13.1 \text { (DE: } 4.1) \\
1.48 \text { (DE: } 1.26) \\
29.1 \text { (DE: } 16.4) \\
135.3 \text { (DE: } 14.2 \text { ) } \\
4.4 \text { (DE: } 3.8) \\
75.3 \text { (DE: } 74.9 \text { ) }\end{array}$ \\
\hline $\begin{array}{l}\text { Estancia hospitalaria } \\
\text { Tiempo promedio (días) }\end{array}$ & 10 (DE: 9) \\
\hline
\end{tabular}

BUN: nitrógeno ureico en sangre; DE: desviación estándar; EPOC: enfermedad pulmonar obstructiva crónica; FEVI: fracción de eyección del ventrículo izquierdo; I.p.m.: latidos por minuto; r.p.m.: respiraciones por minuto; Sat02: saturación arterial de oxígeno.

la ciudad de Bogotá, Colombia. En él se tamizaron 485 pacientes, a 379 de los cuales se les aplicaron las escalas en mención, y se consideró pobre la capacidad de estas para la discriminación del riesgo de muerte durante la estancia hospitalaria ${ }^{10-14}$; resultados que concuerdan con los obtenidos en nuestro estudio.

La mortalidad fue mayor al compararse con la esperada según la razón estandarizada de mortalidad entre 1.29 y 1.41 para los diferentes modelos, asociado a una estancia hospitalaria prolongada, lo cual se atribuye a

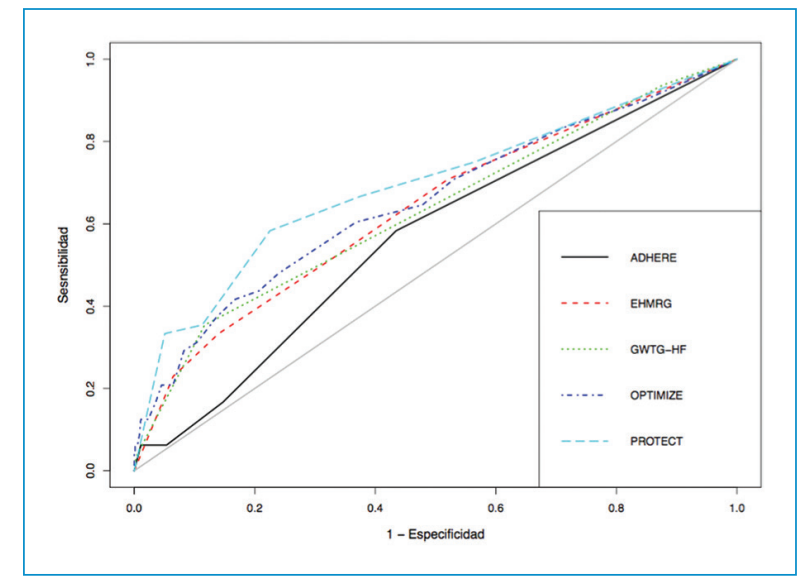

Figura 2. Características operativas con las áreas bajo la curva de las diferentes escalas de riesgo de muerte intrahospitalaria en pacientes con falla cardiaca aguda.

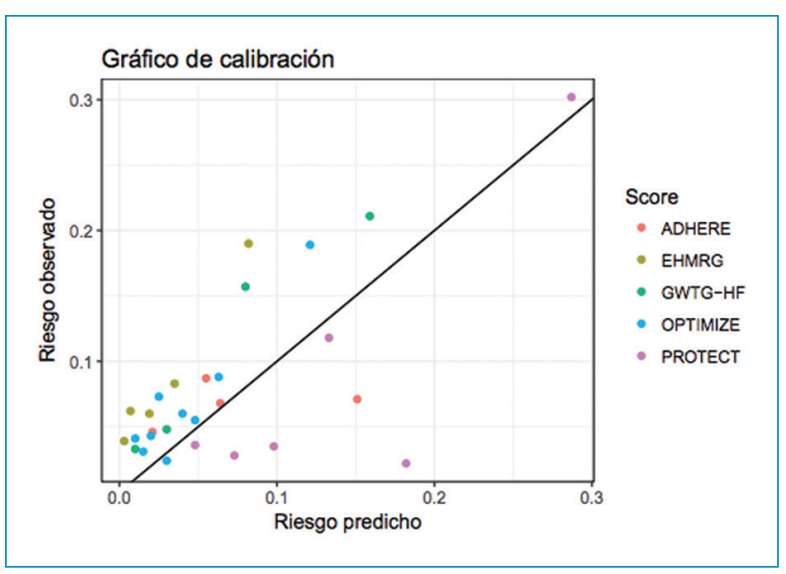

Figura 3. Calibración, riesgo observado y riesgo predicho.

diferencias en nuestra población, que se caracterizó por presentar diversa comorbilidad, fracción de eyección muy comprometida y elevación marcada de los péptidos natriuréticos y de la troponina. La calibración de estas escalas es difícil dada la heterogeneidad de los resultados, por lo que se recomienda aplicarlas solo en poblaciones similares a las utilizadas en los estudios de derivación o en aquellos en los que haya sido evaluada la capacidad predictiva ${ }^{6,13-18}$ (Fig. 3).

Una de las ventajas de este estudio es el gran número de pacientes incluidos, pues la cohorte de falla cardiaca aguda es la más grande en población colombiana publicada hasta el momento; adicionalmente, se evaluaron cinco escalas de predicción de mortalidad en una misma población, con datos suficientes para su cálculo. 
Entre las limitaciones de este estudio están el tipo de diseño retrospectivo, que se haya incluido un solo centro de alta complejidad y de referencia en Colombia para el manejo de falla cardiaca y que la población fuera de alto riesgo con múltiple comorbilidad y en estadios avanzados de falla cardiaca. Así mismo, el número de pacientes es bajo respecto a las cohortes de derivación de los modelos de predicción evaluados y comparados, y la mortalidad global es mayor que la esperada, por lo cual no es posible realizar una validación o recalibración.

En el futuro se requieren estudios multicéntricos, que incluyan instituciones de todos los niveles de atención, con el fin de obtener datos precisos de pacientes con falla cardiaca aguda en Colombia, y de acuerdo con estos, establecer un modelo de predicción de mortalidad intrahospitalaria en nuestra población.

\section{Conclusiones}

Los modelos de predicción de muerte intrahospitalaria en pacientes con falla cardiaca aguda muestran pobre desempeño y baja capacidad de predicción y discriminación en población colombiana, lo cual sugiere el desarrollo de escalas de predicción de mortalidad en pacientes con falla cardiaca aguda específicas para nuestra población.

\section{Agradecimientos}

Los autores agradecen al Hospital Universitario San Ignacio y a la Pontificia Universidad Javeriana.

\section{Conflicto de intereses}

Los autores declaran que no existe conflicto de intereses.

\section{Financiamiento}

Los autores declaran que no existió financiamiento.

\section{Responsabilidades éticas}

Protección de personas y animales. Los autores declaran que para esta investigación no se han realizado experimentos en seres humanos ni en animales.
Confidencialidad de los datos. Los autores declaran que han seguido los protocolos de su centro de trabajo sobre la publicación de datos de pacientes.

Derecho a la privacidad y consentimiento informado. Los autores declaran que en este artículo no aparecen datos de pacientes.

\section{Bibliografía}

1. Jaramillo C, Gómez E, Hernández E, Saldarriaga C, Flórez N, Buitrago R, et al. Consenso colombiano de diagnóstico y tratamiento de la insuficiencia cardiaca aguda. Bogotá: Inversiones Tecnográficas; 2014.

2. De la Fuente-Cid R, Hermida-Ameijeiras A, Pazo-Núñez M, Lado-Lado F. Epidemiología de la insuficiencia cardiaca: proporciones de epidemia. An Med Intern. 2007:24:500-4.

3. Gómez E. Epidemiología de la falla cardiaca e historia de las clínicas de falla cardiaca en Colombia. Rev Colomb Cardiol. 2016;23:6-12.

4. Adams K, Abraham W, Yancy C, Boscardin W. Risk stratification for in-hospital mortality in acutely decompensated heart failure. JAMA 2005;5:572-80.

5. Abraham W, Fonarow G, Albert N, Stough W, Gheorghiade M, Greenberg B, et al. Predictors of in-hospital mortality in patients hospitalized for heart failure. Insights from the Organized Program to Initiate Lifesaving Treatment in Hospitalized Patients With Heart Failure (OPTIMIZE-HF). J Am Coll Cardiol. 2008:5:347-56.

6. Peterson P, Rumsfeld J, Liang L, Albert N, Hernández A, Peterson E, et al. A validated risk score for in-hospital mortality in patients with heart failure from the American Heart Association get with the guidelines program. Circ Cardiovasc Qual Outcomes. 2010;52:25-32.

7. Yancy C, Jessup M, Bozkurt B, Butler J, Casey D, Drazner M, et al. 2013 ACCF/AHA guideline for the management of heart failure: a report of the American College of Cardiology Foundation/American Heart Association Task force on practice guidelines. Circulation. 2013;128:240-327.

8. Lee D, Stitt A, Austin P, Stukel T, Schull M, Chong A, et al. Prediction of heart failure mortality in emergent care. A cohort study. Ann Intern Med. 2012:156:767-75.

9. Oconnor C, Mentz R, Cotter G, Metra M, Cleland J, Davison B, et al. The PROTECT in-hospital risk model: 7-day outcome in patients hospitalized with acute heart failure and renal dysfunction. Eur J Heart Fail. 2012; 14:605-12.

10. Sprockel J, Alfaro L, Cifuentes J, Jiménez M, Barón R, Chaves W. Escalas de estratificación del pronóstico en pacientes con falla cardíaca aguda. Rev Argent Cardiol. 2016;84:574-80.

11. Passantino A, Monitillo $F$, lacoviello M, Scrutinio D. Predicting mortality in patients with acute heart failure: role of risk scores. World $\mathrm{J}$ Cardiol. 2015;7:902-11.

12. McMurray J, Adamopoulos S, Anker S, Auricchio A, Bohm M, Dickstein K, et al. ESC Guidelines for the diagnosis and treatment of acute and chronic heart failure 2012: The Task Force for the Diagnosis and Treatment of Acute and Chronic Heart Failure 2012 of the European Society of Cardiology. Developed in collaboration with the Heart Failure Association (HFA) of the ESC. Eur Heart J. 2012;33:1787-847.

13. Ponikowski $P$, Voors A, Anker $S$, Bueno $H$, Cleland $J$, Coats $A$, et al. ESC Guidelines for the diagnosis and treatment of acute and chronic heart failure. Eur Heart J. 2016;37:2129-200.

14. Ospina A, Gamarra G. Características clínicas y epidemiológicas de la insuficiencia cardiaca en el Hospital Universitario Ramón González Valencia de Bucaramanga, Colombia. Revista Salud UIS. 2004;36:125-31.

15. Auble T, Hsieh M, Gardner W, Cooper G, Stone R, McCausland J, et al. A prediction rule to identify low-risk patients with heart failure. Acad Emerg Med. 2005;12:514-21.

16. Rahimi K, Bennett D, Conrad N, Williams T, Basu J, Dwight J, et al. Risk prediction in patients with heart failure: a systematic review and analysis. JACC Heart Fail. 2014;2:440-6.

17. Pocock S, Ariti C, McMurray J, Maggioni A, Køber L, Squire IB, et al. Predicting survival in heart failure: a risk score based on 39372 patients from 30 studies. Eur Heart J. 2013;34:1404-13.

18. Auble T, Hsieh M, McCausland J, Yealy D. Comparison of four clinical prediction rules for estimating risk in heart failure. Ann Emerg Med. 2007;50:127-35. 